\title{
Mitigation of muscular dystrophy in mice by SERCA overexpression in skeletal muscle
}

\author{
Sanjeewa A. Goonasekera, ${ }^{1}$ Chi K. Lam, ${ }^{2}$ Douglas P. Millay, ${ }^{1}$ Michelle A. Sargent, ${ }^{1}$ Roger J. Hajjar, ${ }^{3}$
} Evangelia G. Kranias, ${ }^{2}$ and Jeffery D. Molkentin ${ }^{1}$

1Department of Pediatrics, University of Cincinnati, Cincinnati Children's Hospital Medical Center, Howard Hughes Medical Institute, Cincinnati, Ohio, USA.
2Department of Pharmacology and Cell Biophysics, University of Cincinnati Medical College, Cincinnati, Ohio, USA. ${ }^{3}$ Cardiovascular Research Center, Mount Sinai School of Medicine, New York, New York, USA.

\begin{abstract}
Muscular dystrophies (MDs) comprise a group of degenerative muscle disorders characterized by progressive muscle wasting and often premature death. The primary defect common to most MDs involves disruption of the dystrophin-glycoprotein complex (DGC). This leads to sarcolemmal instability and $\mathrm{Ca}^{2+}$ influx, inducing cellular necrosis. Here we have shown that the dystrophic phenotype observed in $\delta$-sarcoglycan-null $\left(\mathrm{Sgcd}^{-/-}\right)$ mice and dystrophin mutant $\mathrm{mdx}$ mice is dramatically improved by skeletal muscle-specific overexpression of sarcoplasmic reticulum $\mathrm{Ca}^{2+}$ ATPase 1 (SERCA1). Rates of myofiber central nucleation, tissue fibrosis, and serum creatine kinase levels were dramatically reduced in $\mathrm{Sgcd}^{-/-}$and $\mathrm{mdx}$ mice with the SERCA1 transgene, which also rescued the loss of exercise capacity in $\mathrm{Sgcd}^{-/-}$mice. Adeno-associated virus-SERCA2a (AAV-SERCA2a) gene therapy in the gastrocnemius muscle of $\mathrm{Sgcd}^{-/}$mice mitigated dystrophic disease. SERCA1 overexpression reversed a defect in sarcoplasmic reticulum $\mathrm{Ca}^{2+}$ reuptake that characterizes dystrophic myofibers and reduced total cytosolic $\mathrm{Ca}^{2+}$. Further, SERCA1 overexpression almost completely rescued the dystrophic phenotype in a mouse model of MD driven solely by $\mathrm{Ca}^{2+}$ influx. Mitochondria isolated from the muscle of SERCA1-Sgcd ${ }^{-/}$mice were no longer swollen and calpain activation was reduced, suggesting protection from $\mathrm{Ca}^{2+}$-driven necrosis. Our results suggest a novel therapeutic approach using SERCA1 to abrogate the altered intracellular $\mathrm{Ca}^{2+}$ levels that underlie most forms of $\mathrm{MD}$.
\end{abstract}

\section{Introduction}

MD broadly encompasses a diverse group of genetic disorders that result in progressive muscle wasting and premature death (1). The majority of genes identified in patients with MD appear to contribute to or affect the multi-protein sarcolemmal-spanning dystrophin-glycoprotein complex (DGC), which is critical for maintaining integrity of the membrane and the proper activity of signaling complexes and channels (2). A disruption in the DGC is hypothesized to promote direct $\mathrm{Ca}^{2+}$ influx and/or abnormal cytosolic $\mathrm{Ca}^{2+}$ homeostasis, leading to increased vulnerability of myofibers to necrosis (3). However, direct measurement of intracellular $\mathrm{Ca}^{2+}$ concentration in dystrophic muscle has revealed conflicting results as to whether or not $\mathrm{Ca}^{2+}$ is elevated at baseline (4-9). Despite the lack of consensus with direct physical measurements, numerous studies have demonstrated that increased cytosolic $\mathrm{Ca}^{2+}$ activity can cause or enhance the dystrophic phenotype in muscle (10-12). For example, overexpression of transient receptor potential canonical (TRPC3) in skeletal muscle, which facilitates $\mathrm{Ca}^{2+}$ and $\mathrm{Na}^{+}$influx, directly induced the entire dystrophic phenotype without destabilizing the sarcolemma (10). Similarly, blunting of select $\mathrm{Ca}^{2+}$-mediated disease processes can reduce the dystrophic phenotype of muscle in various mouse models of $\operatorname{MD}(10,13,14)$.

In skeletal muscle, the sarcoplasmic reticulum $\mathrm{Ca}^{2+}$ ATPase 1 (SERCA1) isoform is dominantly responsible for $\mathrm{Ca}^{2+}$ reuptake into the sarcoplasmic reticulum (SR) during excitation contraction (EC) coupling (15). Interestingly, a reduction in SERCA activity has been observed in dystrophic muscle (16-18), which is likely responsible for aspects of defective $\mathrm{Ca}^{2+}$ handling in $\mathrm{MD}$, leading

Conflict of interest: The authors have declared that no conflict of interest exists. Citation for this article: J Clin Invest. 2011;121(3):1044-1052. doi:10.1172/JCI43844. to greater cytoplasmic levels and cellular necrosis through calpain activation and mitochondrial permeability transition pore (MPTP) formation $(3,7,12,14)$. Here we hypothesized that $\mathrm{Ca}^{2+}$ is a finalcommon pathway for mediating cellular necrosis and end-stage disease across most of the MDs. Indeed, we show that SERCA1 overexpression in skeletal muscle dramatically attenuates manifestations of dystrophic disease in a Duchenne and limb-girdle model of $\mathrm{MD}$ in the mouse.

\section{Results}

Generation of SERCA1 Tg mice. Increased ryanodine receptor (RyR) $\mathrm{Ca}^{2+}$ leak and reduced SR $\mathrm{Ca}^{2+}$ cycling are thought to contribute to MD pathogenesis $(16,18-20)$. Moreover, diminished SERCA1 activity characteristic of dystrophic muscle should exacerbate a $\mathrm{Ca}^{2+}$ overload problem associated with an unstable sarcolemma (3, 21, 22). In an attempt to restore and enhance SERCA1 activity and $\mathrm{SR} \mathrm{Ca}^{2+}$ reuptake, we generated a series of Tg mice using the skeletal muscle-specific skeletal $\alpha$-actin promoter to drive SERCA1. While 12 lines were generated and partially analyzed for protein expression and phenotypic effects, 1 line was selected for in-depth analysis. Western blotting from 1 line showed 2- to 4-fold overexpression of SERCA1 protein across the quadriceps, gastrocnemius, diaphragm, and soleus, while no expression was observed in the heart (Figure 1A). H\&E- and Masson's trichrome-stained histological sections showed no pathological features in the muscles of SERCA1 Tg mice compared with non-Tg littermates, although fiber cross-sectional areas were slightly decreased (Figure 1B). Next, electrically evoked $\mathrm{Ca}^{2+}$ transients were assessed in acutely isolated flexor digitorum brevis (FDB) fibers to determine whether SERCA1 overexpression promotes SR loading and enhanced $\mathrm{Ca}^{2+}$ clearance. As predicted, peak $\mathrm{Ca}^{2+}$ transient amplitudes were sig- 
A

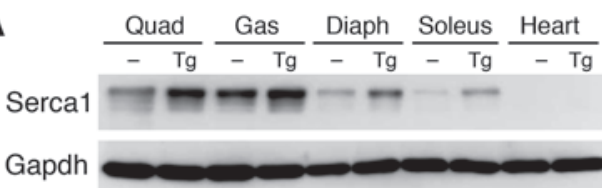

B
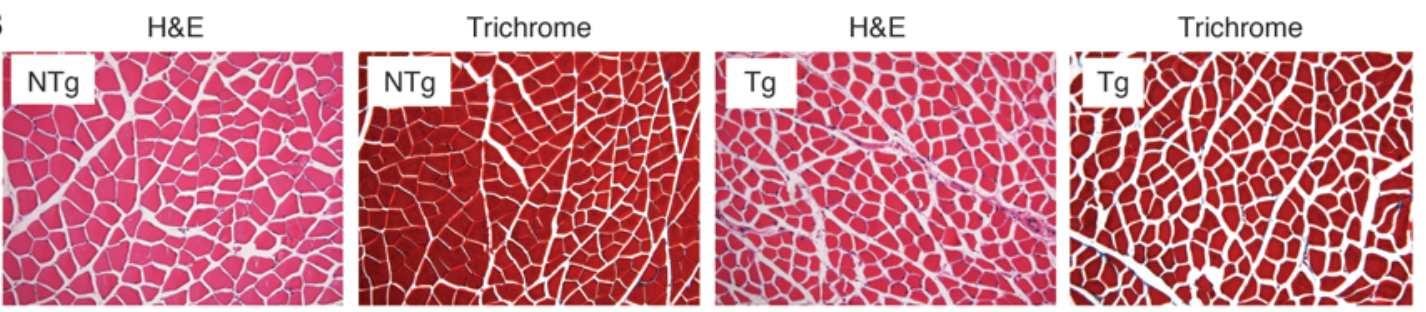

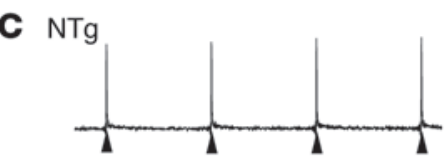

Electrical stimulation

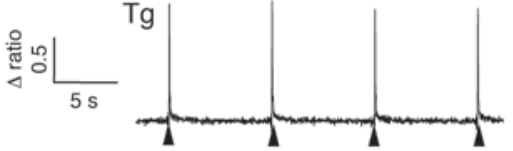

Electrical stimulation
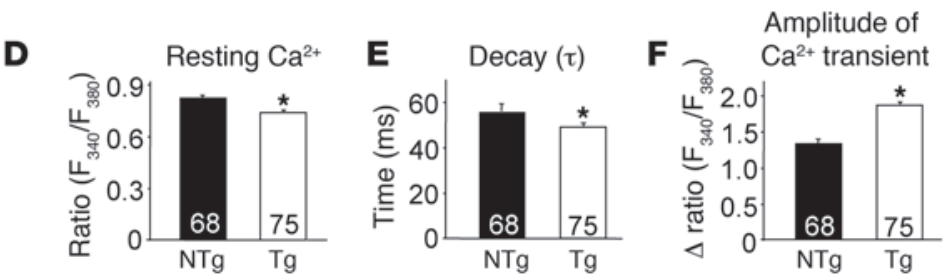

Figure 1

Overexpression of SERCA1 in skeletal muscle enhances $\mathrm{Ca}^{2+}$ cycling during EC coupling. (A) Western blot analysis for SERCA1 expression in different muscle groups isolated from non- $\mathrm{Tg}(\mathrm{NTg})$ and SERCA1 $\mathrm{Tg}(\mathrm{Tg})$ mice at 3 months of age. Quad, quadriceps; Gas, gastrocnemius; Diaph, diaphragm. (B) H\&E and Masson's trichrome sections of quadriceps. Original magnification, $\times 200$. (C) Representative traces of $F_{340} / F_{380}$ fluorescence ratio recordings from single FDB myofibers isolated from NTg and SERCA1 Tg mice in response to electrical stimulation. (D) Resting $\mathrm{Ca}^{2+}$ ratio, $(\mathbf{E})$ time constant of decay $(\tau)$, and $(\mathbf{F})$ peak $\mathrm{Ca}^{2+}$ transient amplitude in isolated myofibers from the indicated genotypes. ${ }^{*} P<0.05$ compared with NTg mice; $n=$ total number of fibers recorded from 4 animals in each genotype shown in the graphs, D-F.

nificantly increased and the time to decay of the $\mathrm{Ca}^{2+}$ transient was reduced (Figure 1, C, E, and F). Interestingly, the resting $\mathrm{Ca}^{2+}$ ratio was also significantly decreased in FDB fibers isolated from SERCA1 Tg mice compared with non-Tg littermates (Figure 1D). Taken together, these results demonstrate that overexpression of SERCA1 in skeletal muscle enhances $\mathrm{Ca}^{2+}$ clearance and cycling. However, expression levels of critical SR $\mathrm{Ca}^{2+}$ handling proteins were not significantly changed by the SERCA1 transgene, as assessed by quantitative Western blotting (Supplemental Figure 1; supplemental material available online with this article; doi:10.1172/JCI43844DS1).

SERCA1 overexpression ameliorates $M D$ in $\delta$-sarcoglycan null mice. To assess the potential beneficial effect of SERCA1 overexpression in the context of MD, we crossed the SERCA1 transgene into the $\delta$-sarcoglycan null $\left(\mathrm{Sgcd}^{-/-}\right)$genetic background, the latter of which is a mouse model with fulminant dystrophic disease $(23,24)$. The presence of the SERCA1 transgene resulted in a remarkable reduction in histopathology in $\mathrm{Sgcd^{-/ }}$ mice at 6 weeks, 3 months, and 6 months of age, showing severe reductions in myofibers with centrally located nuclei and overall rates of fibrosis compared with Sgcd ${ }^{-/}$only controls (Figure 2, A-F). In addition, serum creatine kinase (CK) levels were significantly reduced in $\mathrm{Sgcd}^{-/}$mice with the SERCA1 transgene compared with $\mathrm{Sgcd}^{-/-}$littermates (Fig- ure $2 \mathrm{H}$ ). Associated with this improvement in histopathology, $S g c d^{-1-}$ mice with the SERCA1 transgene showed a restoration in their ability to run on a treadmill comparable to WT control mice, while $\mathrm{Sgcd}^{-/-}$mice alone were severely compromised (Figure $2 \mathrm{G}$ ). We also investigated whether sarcolemmal rupture rates and subsequent Evans blue dye (EBD) uptake were affected by the SERCA1 transgene in Sgcd ${ }^{--}$mice. Normally, muscle fibers from $S g c d^{-/-}$mice show uptake of EBD after systemic injection due to membrane breaks, which was observed in the quadriceps, gastrocnemius, tibialis anterior (TA), and diaphragm from control null mice only (Figure 2, I and J). However, the presence of the SERCA1 transgene uniformly reduced total EBD uptake in $\mathrm{Sgcd}^{-/-}$mice by 3 - to 5 -fold (Figure 2, I and J). We interpret this reduction in EBD uptake with the SERCA1 transgene to result from enhanced $\mathrm{Ca}^{2+}$ reuptake, which likely reduces membrane and mitochondrial reactive oxygen species generation that could secondarily diminish membrane stress and a presumed feed-forward mechanism of disease (see discussion). The soleus muscle was not differentially affected, presumably because it is a postural muscle that is always under tension/use (Figure 2J).

SERCA1 overexpression ameliorates $M D$ in $m d x$ mice. The mouse model of Duchenne MD with defective dystrophin expression results from the $m d x$ mutation. This model, which corresponds 
A
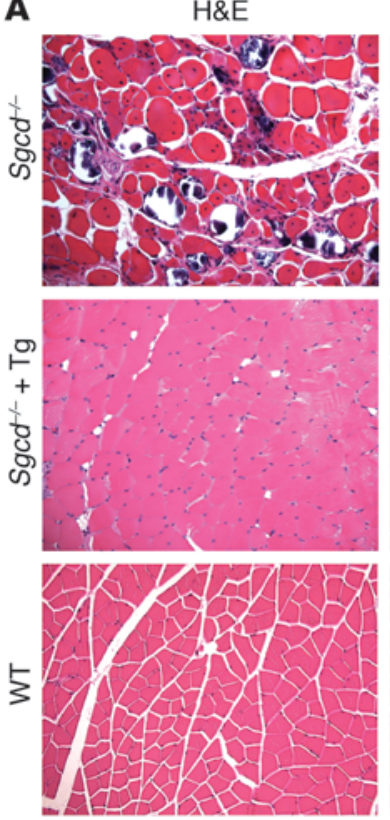

H

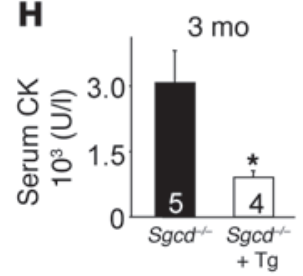

Trichrome
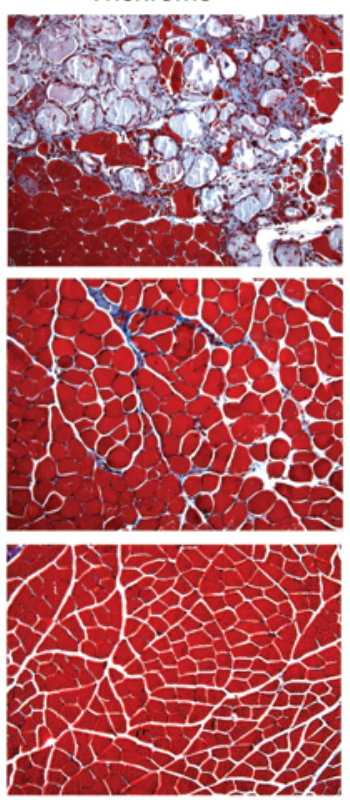

I

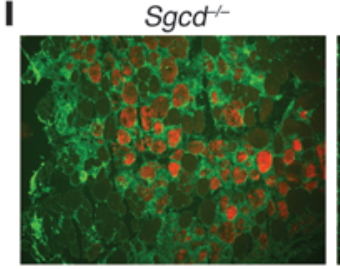

B

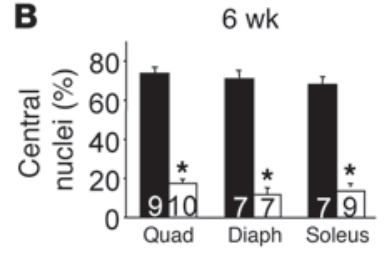

D

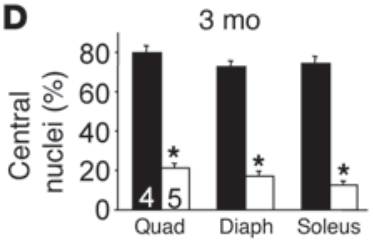

F

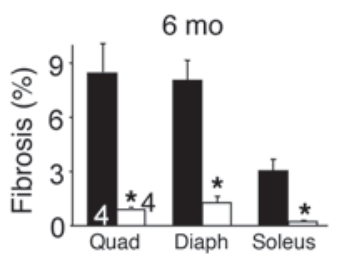

C

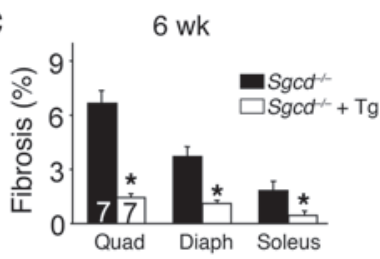

E

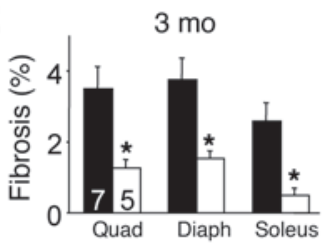

G

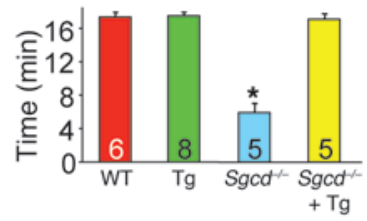

Figure 2

SERCA1 mitigates biochemical and histological features of MD in Sgcd ${ }^{-1-}$ mice. (A) H\&E- and Masson's trichrome-stained sections of quadriceps from WT, Sgcd ${ }^{-1-}$, and $S g c d^{-1-S E R C A 1 ~ T g ~ m i c e ~ a t ~} 3$ months of age. Original magnification, $\times 200$. (B and D) Percentage of myofibers with centrally located nuclei in Sgcd ${ }^{-/-}$and $S g c d^{-1-S E R C A 1 ~ T g ~ m i c e ~ a t ~} 6$ weeks and 3 months of age. (C, E, and F) Interstitial fibrosis in muscle histological sections analyzed using metamorph analysis software in $\mathrm{Sgcd}^{-/-}$and $\mathrm{Sgcd}^{-1-}$-SERCA1 Tg mice at 6 weeks, 3 months, and 6 months of age. Number of mice used for quantitation is shown in the graphs. (G) Time to fatigue in minutes with forced treadmill running in the indicated groups of mice. (H) Quantitation of serum CK levels in Sgcd ${ }^{-1-}$ and Sgcd ${ }^{-1-S E R C A 1 ~ T g ~ m i c e ~ a t ~} 3$ months of age. (I) Representative immunofluorescence images of EBD uptake in histological sections from quadriceps of 3-month-old mice subjected to running for 2 days in the presence of EBD. Membranes are stained green while EBD-positive fibers are in red. Original magnification, $\times 100$. (J) Quantitation of total EBD fibers in quadriceps, gastrocnemius, TA, diaphragm, and soleus from at least 3 mice in each genotype. ${ }^{*} P<0.05$ versus $S g c d^{-1-}$. Number of mice used is shown in each of the graphs.

to the most prevalent form of human $\mathrm{MD}$, is typically less severe, and mice can live a near normal lifespan compared with $\mathrm{Sgcd}^{-1}$ mice, which manifest more severe disease with premature lethality $(23,24)$. Here we crossed the SERCA1 transgene into the $m d x$ genetic background, and similarly to what we observed in the Sgcd ${ }^{-1}$ - background, both histological and biochemical markers of MD were dramatically diminished (Figure 3A). At both 6 weeks and 3 months of age, myofiber central nucleation was profoundly reduced in the quadriceps, diaphragm, and soleus (Figure 3, B and D). In fact, at 6 weeks of age, myofiber central nucleation was nearly extinguished in all 3 muscles analyzed (Figure 3B). While fibrosis typically only becomes prominent in older $m d x$ mice, even the mild degree of tissue fibrosis that begins to develop at 6 weeks and 3 months of age was significantly reduced in skeletal muscle of SERCA1/mdx mice (Figure 3, C and E). Finally, serum CK levels at 3 months of age were also reduced by approximately 4 -fold in SERCA1/ $m d x$ mice when compared with $m d x$ mice (Figure $3 F)$. These results further support the conclusion that enhanced
SERCA1 expression can attenuate histopathology and biochemical features of MD in mouse models of disease.

SERCA1 overexpression ameliorates MD in TRPC3-overexpressing Tg mice. We demonstrated that skeletal muscle-specific overexpression of the ion channel TRPC3 was sufficient to induce skeletal muscle disease that was entirely consistent with MD (10). TRPC channels are thought to contribute to $\mathrm{Ca}^{2+}$ influx in $\mathrm{MD}$ associated with destabilization of the sarcolemma. Indeed, overexpression of a dominant negative TRPC6 mutant protein in skeletal muscle blocked $\mathrm{Ca}^{2+}$ leak activity of the sarcolemma and significantly reduced MD disease manifestations in $m d x$ and $S g c d^{-/-}$mice (10). Here we intercrossed TRPC3- and SERCA1-overexpressing Tg mice to more directly examine the " $\mathrm{Ca}^{2+}$ hypothesis," since the only disease-inducing effect associated with the TRPC 3 transgene is $\mathrm{Ca}^{2+}$ influx. As predicted, H\&E staining of quadriceps and soleus muscle showed dramatically less tissue histopathology at 3 months of age in SERCA1/TRPC3 Tg mice compared with TRPC $3 \mathrm{Tg}$ mice (Figure 4A). For example, quantitation of centrally located myo- 
A
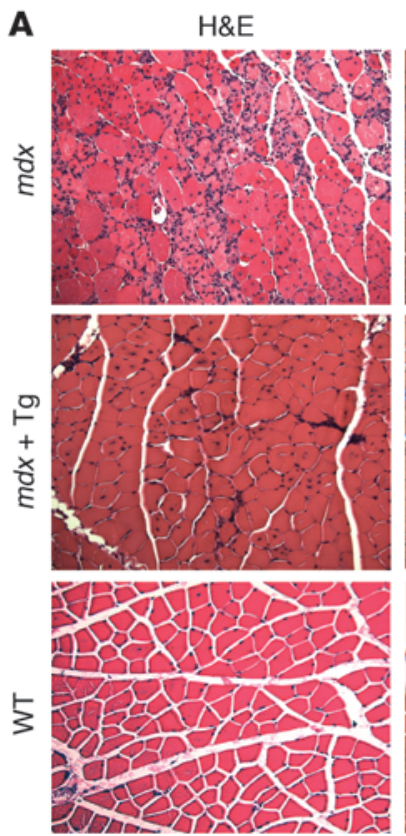

Trichrome
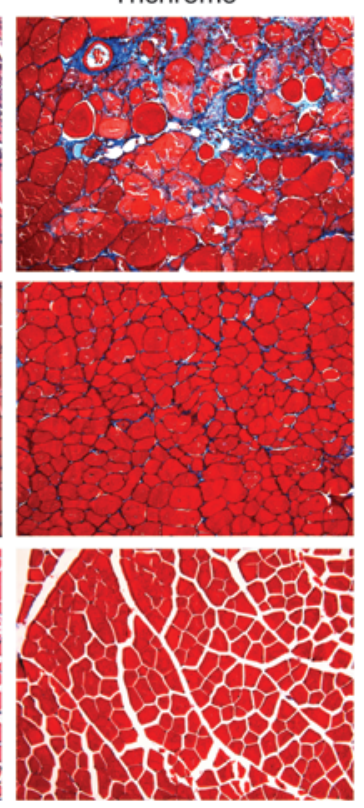

B

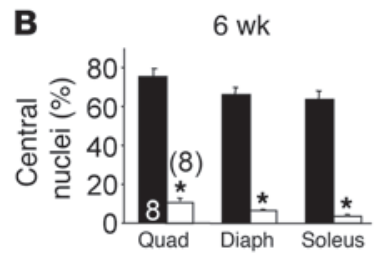

D
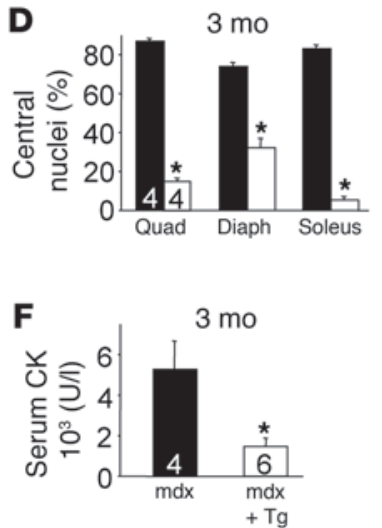

C

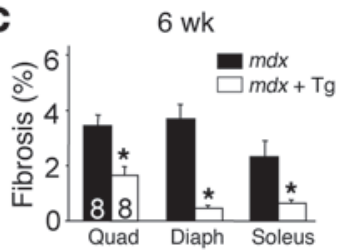

E

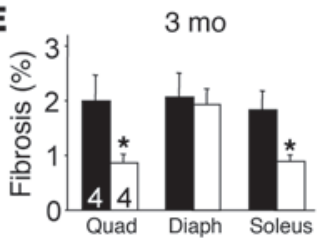

\section{Figure 3}

SERCA1 mitigates biochemical and histological features of MD in $m d x$ mice. (A) H\&E- and Masson's trichrome-stained histological sections from quadriceps of WT, $m d x$, and $m d x$-SERCA1 Tg mice at 3 months of age. Original magnification, $\times 200$. (B and D) Percentage of myofibers with centrally located nuclei in $m d x$ and $m d x$-SERCA1 Tg mice at 6 weeks and 3 months of age. (C and E) Interstitial fibrosis in muscle histological sections analyzed using metamorph analysis software in $m d x$ and $m d x$-SERCA1 Tg mice at 6 weeks and 3 months of age. Number of mice used for quantitation is shown in the graphs. (F) Quantitation of serum CK levels in $m d x$ and $m d x-S E R C A 1$ Tg mice at 3 months of age. ${ }^{\star} P<0.05$ versus $m d x$. Number of mice used is shown in the graphs.

fibers showed significant reductions in SERCA1/TRPC3 Tg mice compared with TRPC3 Tg mice (Figure 4B). These results further support the hypothesis that SERCA1 overexpression is protective by augmenting SR $\mathrm{Ca}^{2+}$ handling and counteracting the effect of increased membrane $\mathrm{Ca}^{2+}$ leak in MD.

Enhanced SR Ca ${ }^{2+}$ uptake in SERCA1-overexpressing mice. To further investigate the mechanism whereby SERCA1 overexpression can protect against $\mathrm{MD}$, we directly measured $\mathrm{SR} \mathrm{Ca}^{2+}$ uptake in lysates from muscle of the relevant genotypes. As predicted, SR $\mathrm{Ca}^{2+}$ uptake was approximately 2 -fold higher in $\mathrm{WT}\left(\mathrm{Sgcd}^{+/+}\right)$mice containing the SERCA1 transgene compared with mice without the SERCA1 transgene (Figure 5, A and C). Consistent with past observations in dystrophic myofibers/myotubes, maximal SR $\mathrm{Ca}^{2+}$

\section{Figure 4}

SERCA1 mitigates histological features of MD in TRPC3 Tg mice. (A) Representative histological H\&E stain of quadriceps from TRPC3 Tg and TRPC3/SERCA1 double-Tg at 3 months of age. Original magnification, $\times 200$. (B) Percentage of myofibers with centrally located nuclei in quadriceps and soleus from TRPC $3 \mathrm{Tg}$ and TRPC3/SERCA1 double-Tg mice. At least 3 mice from each genotype were used. ${ }^{\star} P<0.05$ versus TRPC3 TG. Number of mice used is shown in the graph. uptake was significantly reduced in the $S g c d^{-/-}$mice compared with WT controls $\left(\mathrm{Sgcd}^{+/+}\right)$(Figure 5C). More importantly, SR Ca ${ }^{2+}$ uptake in $\mathrm{Sgcd}^{-/-} \mathrm{Tg}$ mice was 3-fold greater than in $\mathrm{Sgcd}^{-/-}$mice and activity was restored to levels higher than even that in WT controls (Figure 5, B and C). The calculated $\mathrm{EC}_{50}$ value (pCa) was not significantly different in any of the groups, suggesting that increased expression of SERCA1 did not change its affinity for $\mathrm{Ca}^{2+}$ (data not shown). Collectively, these results indicate that SERCA1 overexpression dramatically enhances $\mathrm{SR} \mathrm{Ca}^{2+}$ reuptake capacity and corrects a deficit observed in $\mathrm{Sgcd}^{-/-}$myofibers.

SERCA1 overexpression restores EC-coupling defects observed in dystrophic myofibers. Multiple studies have shown deficits in global EC-coupling in dystrophic muscle fibers (25-27). These include a

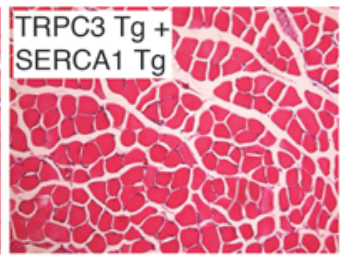

B

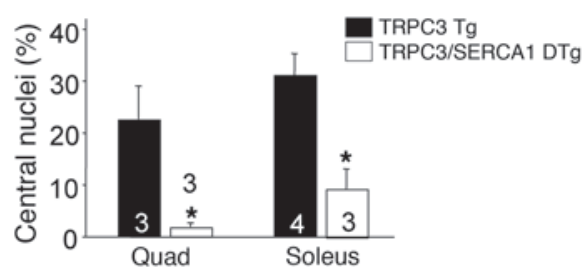



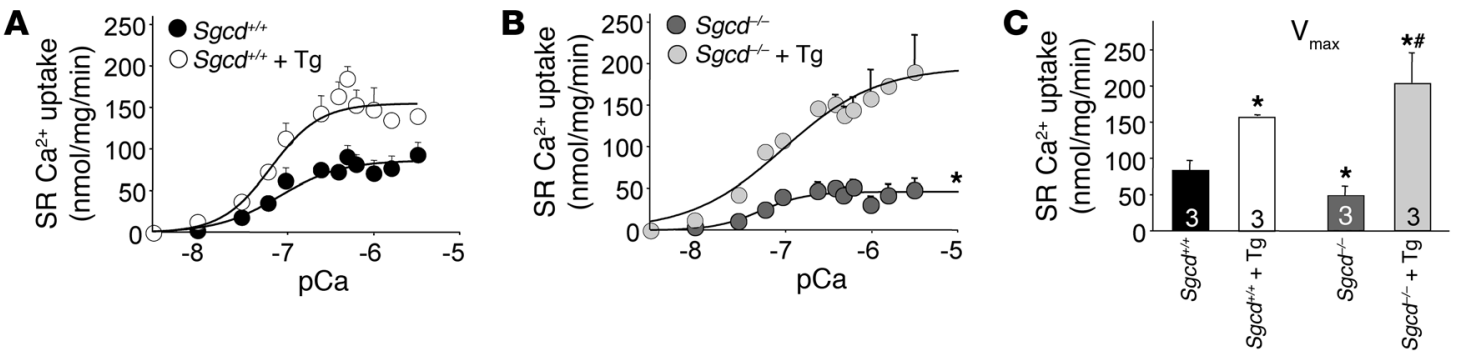

\section{Figure 5}

SERCA1 enhances SR Ca ${ }^{2+}$ uptake in $S g c d^{-/-}$mice. (A) Average mean values of SR Ca ${ }^{2+}$ uptake in $S g c d^{+/+}(\mathrm{WT})$ and $S g c d^{+/+}-\mathrm{SERCA} 1 \mathrm{Tg}$ mice as a function of $\mathrm{pCa}^{2+}$. (B) Average mean values of $\mathrm{SR} \mathrm{Ca}{ }^{2+}$ uptake in $\mathrm{Sgcd}^{-/-}$and $\mathrm{Sgcd}^{-/-} \mathrm{SERCA1} \mathrm{Tg}$ mice as a function of pCa ${ }^{2+}$. (C) Mean maximal velocity determined for each genotype in the SR Ca ${ }^{2+}$ uptake measurements. ${ }^{*} P<0.05$ compared with $S g c d^{+/+;}{ }^{*} P<0.05$ compared with $\mathrm{Sgcd}^{-1-}$. Number of mice used is shown in the graph.

significant reduction in the peak amplitude of the $\mathrm{Ca}^{2+}$ transient and diminished $\mathrm{Ca}^{2+}$ reuptake into the SR. Here we investigated whether enhancing SR Ca ${ }^{2+}$ uptake with SERCA1 overexpression could ameliorate the defects in global EC coupling observed in dystrophic myofibers. We assessed electrically evoked $\mathrm{Ca}^{2+}$ transients in acutely isolated individual FDB fibers in $\mathrm{Sgcd}^{+/+}$and $S g \mathrm{gd}^{-/}$mice with and without the SERCA1 transgene. Interestingly, the peak amplitude of the $\mathrm{Ca}^{2+}$ transient was increased in WT $\left(\mathrm{Sgcd}^{+/+}\right)$myofibers with the SERCA1 transgene compared with WT alone (Figure 6, A, B, and E). Consistent with results in the literature, the peak amplitude of the $\mathrm{Ca}^{2+}$ transient was reduced in $\mathrm{Sgcd}^{-/-}$myofibers compared with WT fibers, but this decrease was prevented with the SERCA1 transgene (Figure 6, C-E). SR $\mathrm{Ca}^{2+}$ load assessed with 4-chloro-m-cresol (4-CMC, to release SR $\mathrm{Ca}^{2+}$ stores) was not significantly different between $\mathrm{Sgcd}^{-1-}$ and WT myofibers (Figure 6G). However, the SERCA1 transgene did significantly elevate SR $\mathrm{Ca}^{2+}$ load in WT myofibers above WT lev- els, but not in $S g c d^{-/-}$myofibers (Figure 6G). More importantly, the reuptake phase of the 4-CMC-induced $\mathrm{Ca}^{2+}$ profile and the decay time of the $\mathrm{Ca}^{2+}$ transient were each significantly prolonged in myofibers from $\mathrm{Sgcd}^{-1-}$ mice, and this defect was corrected with the SERCA1 transgene (Figure 6, C, D, and F). Similarly, FDB myofibers from $m d x$ mice also showed a reduction in the amplitude of the $\mathrm{Ca}^{2+}$ transient and an increase in relaxation time, parameters that were again corrected by the SERCA1 transgene (Figure 6, H and I). Taken together, these results demonstrate that dystrophic myofibers have significant defects in $\mathrm{SR} \mathrm{Ca}^{2+}$ handling, which can be corrected by SERCA1 expression.

SERCA1 overexpression reverses mitochondrial swelling in dystrophic muscle. We recently demonstrated that mitochondria isolated from dystrophic skeletal muscle of $\mathrm{Sgcd}^{-/-}$mice are swollen at baseline and that this swelling can lead to myofiber necrosis (14). Indeed, prevention of $\mathrm{Ca}^{2+}$-mediated mitochondrial swelling and myofiber necrosis by deletion of cyclophilin D or use of Debio-025 in
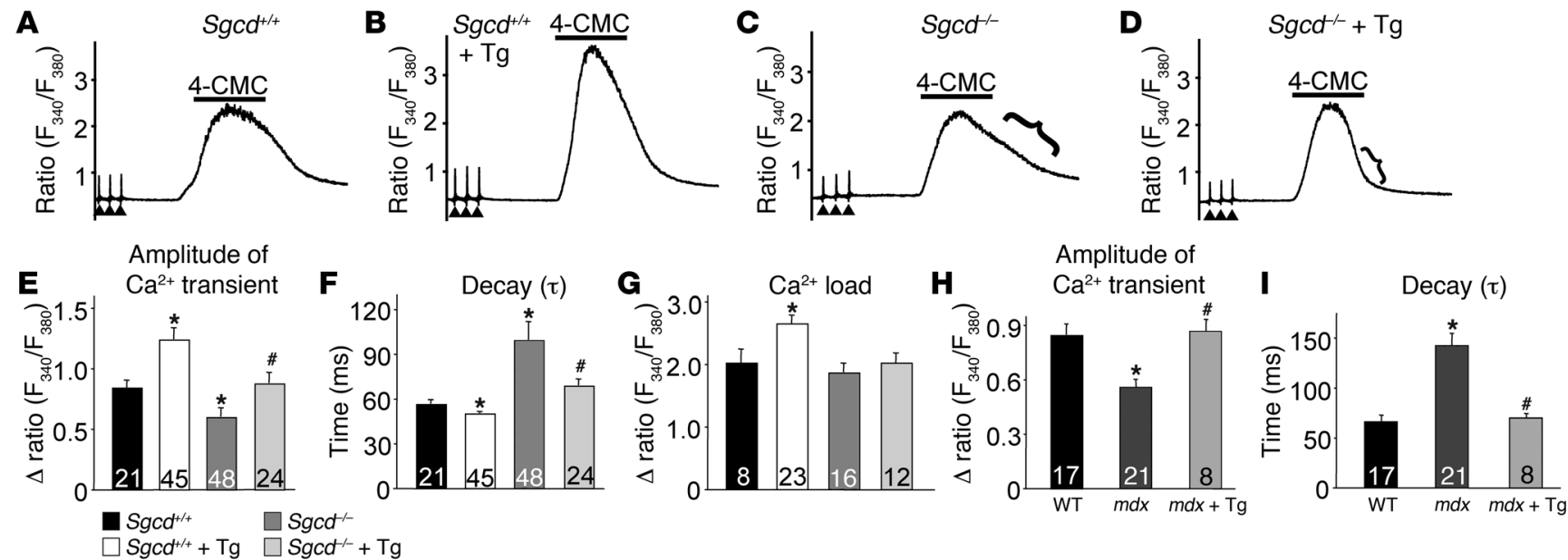

Figure 6

SERCA1 overexpression enhances EC coupling and $\mathrm{Ca}^{2+}$ clearance in Sgcd $\mathrm{Cl}^{-/}$and $\mathrm{mdx}$ myofibers. (A-D) Representative traces of changes in ratiometric fluorescence ratios in acutely isolated FDB fibers from $\mathrm{Sgcd}^{-1-}$ mice in response to electrical and chemical stimulation over time. The brackets in $\mathbf{C}$ and $\mathbf{D}$ show the $\mathrm{Ca}^{2+}$ reuptake characteristics. (E) Amplitude of the $\mathrm{Ca}^{2+}$ transient after electrical twitch stimulation in the indicated genotypes shown in the legend next to $\mathbf{F}$ (applies to $\mathbf{E}-\mathbf{G})$. (F) Time constant of decay $(\tau)$ and $(\mathbf{G})$ maximal response to 4-CMC in the indicated genotypes as assessed with Fura-2 ratio analysis. ${ }^{*} P<0.05$ versus $\mathrm{Sgcd}^{+/+} ;{ }^{*} P<0.05$ versus $\mathrm{Sgcd}^{-/-}$. Number of fibers used in $\mathbf{E}-\mathbf{G}$ is shown in the graphs. (H) Amplitude of the $\mathrm{Ca}^{2+}$ transient after electrical twitch stimulation in FDB myofibers from $m d x$ and control mice as indicated. ${ }^{*} P<0.05$ versus WT; ${ }^{\#} P<0.05$ versus $m d x$. (I) Time constant of decay $(\tau)$ of the $\mathrm{Ca}^{2+}$ transient in FDB myofibers in the indicated $m d x$ and control groups. ${ }^{*} P<0.05$ versus $\mathrm{WT} ;{ }^{\#} P<0.05$ versus $m d x$. 
A

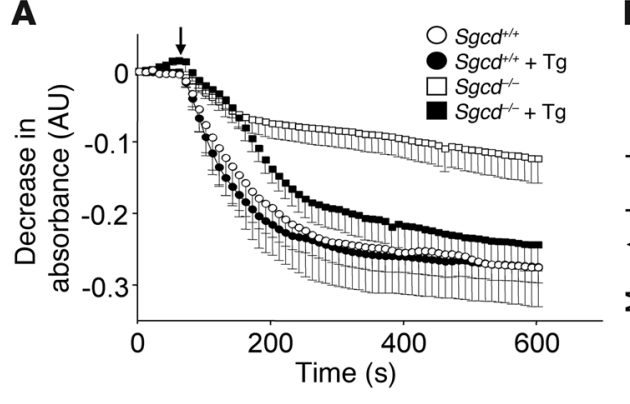

B

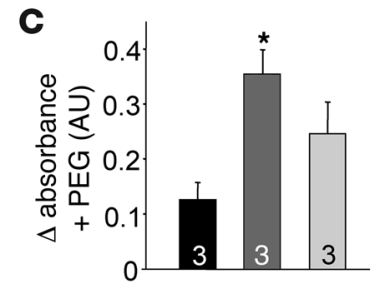

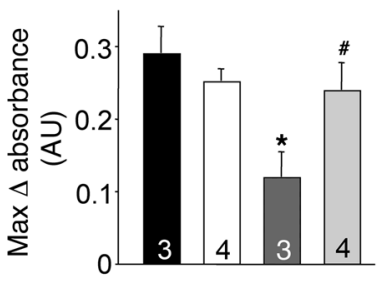

Figure 7

SERCA1 overexpression protects against $\mathrm{Ca}^{2+}$-induced disease indexes in skeletal muscle of $\mathrm{Sgcd}^{-1-}$ mice. (A) Mitochondrial swelling after $\mathrm{Ca}^{2+}$ addition (arrow) as a function of increased light scattering (decreased absorbance) from muscle purified mitochondria from Sgcd ${ }^{+/+}, \mathrm{Sgcd}^{+/+_{-}}$ SERCA1 Tg, Sgcd ${ }^{-1-}$, and Sgcd ${ }^{-1-}$-SERCA1 Tg mice. (B) Average maximal change in absorbance in response to external $\mathrm{Ca}^{2+}$ in purified mitochondria from the indicated groups. ${ }^{*} P<0.05$ compared with $S g c d^{+/+}$; $P<0.05$ versus $\mathrm{Sgcd}^{-1-}$. (C) Change in absorbance in response to PEG to show degree of mitochondrial shrinkage in purified mitochondria. ${ }^{*} P<0.05$ compared with $S g c d^{+++}$. (D) Calpain enzymatic activity in skeletal muscle of the indicated groups ${ }^{*} P<0.05$ compared with $\mathrm{Sgcd}^{+/+} ; \# P<0.05$ versus $\mathrm{Sgcd}^{-/}$. Number of mice used is shown in the bars of each panel. mice (both of which inhibit MPTP) significantly reduced MD in $S g c d^{-/}$and $m d x$ mice (14). Here we hypothesized that the SERCA1 transgene would reduce $\mathrm{Ca}^{2+}$ overload and spare mitochondria from swelling and induction of myofiber necrosis. Mitochondrial swelling was measured in purified preparations in vitro after isolation from muscle; thereafter swelling/shrinking was assessed with exogenous $\mathrm{Ca}^{2+}$ or PEG3350, respectively. As we have previously observed, mitochondria from $\mathrm{Sgcd}^{-/-}$muscle showed a significantly reduced capacity to swell upon $\mathrm{Ca}^{2+}$ addition compared with $\mathrm{Sgcd}^{+/+}$mitochondria given their already swollen state, although shrinkage was enhanced (Figure 7, A-C). As predicted, this swollen state of mitochondria from $\mathrm{Sgcd}^{-/-}$muscle was rescued by the SERCA1 Tg (Figure 7, A-C). $\mathrm{Ca}^{2+}$ overload in dystrophic skeletal muscle is associated with calpain activation, which can lead to myofiber degeneration and cellular necrosis (12). We observed an increase in calpain enzymatic activity in muscle from $\mathrm{Sgcd}^{-/-}$mice, and importantly, this increase was significantly reduced by the presence of the SERCA1 transgene (Figure 7D). Collectively, these results again demonstrate that the SERCA1 transgene corrects $\mathrm{Ca}^{2+}$ overload, which likely protects the myofibers from cell death by diminishing mitochondrial swelling and calpain activation, reducing myofiber necrosis.

SERCA2 a gene therapy in Sgcd ${ }^{-/}$mice mitigates dystrophic disease. The results presented to this point suggest that SERCA overexpression could serve as a "universal" therapy for many diverse forms of MD that are associated with a $\mathrm{Ca}^{2+}$ imbalance or a leaky plasma membrane. Hence, a gene therapeutic strategy to overexpress SERCA protein in dystrophic skeletal muscle could be an exciting new approach to consider to treat patients. Here we injected 3-day-old $\mathrm{Sgcd}^{-/-}$pups with adeno-associated virus 9-SERCA2a (AAV9-SERCA2a) in the left gastrocnemius or AAV9-GFP (control) in the right gastrocnemius with $10^{10}$ viral particles each. The AAV9 serotype yields robust expression in muscle, and the SERCA2a protein functions very similarly to SERCA1. Mice were then harvested 6 weeks later for full histological assessment of muscle disease and expression of GFP. GFP expression was observed throughout the gastrocnemius of $\mathrm{Sgcd}^{-/-}$mice (greater than $90 \%$ of the fibers; data not shown), and tissue pathology was fulminant in the control injected muscle, as is typically observed (Figure 8A). However, expression of SERCA2a by AAV-mediated gene therapy dramatically attenuated dystrophic disease in the injected gastrocnemius, showing 8-fold less central nucleation and preserved cellular architecture with little signs of necrosis (Figure 8, A and B). Thus, AAV-SERCA gene therapy is an exciting new avenue to potentially pursue for treating human dystrophic or myopathic diseases.

\section{Discussion}

Loss of sarcolemmal stability associated with mutations in genes comprising or affecting components of the DGC has been established as a key insult leading to the development of $\operatorname{MD}(22,28$, 29). Dysregulated and enhanced $\mathrm{Ca}^{2+}$ influx across an unstable sarcolemma has been proposed to directly program myofiber degeneration and produce disease $(3,21)$. For example, elevated cytosolic $\mathrm{Ca}^{2+}$ can enhance $\mathrm{Ca}^{2+}$-dependent protease activity (calpain) and mitochondrial $\mathrm{Ca}^{2+}$ overload-induced cell death as well as increase reactive oxygen and nitrogen species to mediate myofiber death (3, $7,11,21,30)$. In support of this hypothesis, a number of groups have reported abnormally high levels of intracellular $\mathrm{Ca}^{2+}$ in dystrophic myotubes and adult myofibers isolated from dystrophic muscle cells (4-6). However, other groups failed to observe elevated free cytosolic $\mathrm{Ca}^{2+}$ in similar muscle preparations $(8,9)$. By comparison, studies focusing on cardiomyopathy in sarcoglycan-deficient animals are also consistent with the $\mathrm{Ca}^{2+}$ hypothesis of disease, as the L-type $\mathrm{Ca}^{2+}$ inhibitor verapamil reduced some aspects of heart disease in this mouse model, as did gene therapy with a dominant negative phospholamban protein (enhances SERCA2a function and $\mathrm{Ca}^{2+}$ clearance) in a hamster model $(31,32)$.

More recent evidence in genetically modified mouse models has further buttressed the $\mathrm{Ca}^{2+}$ hypothesis of disease in MD. For example, overexpression of the $\mathrm{Ca}^{2+} / \mathrm{Na}^{+}$permeable TRPC 3 channel in skeletal muscle of Tg mice produced all the histological and biochemical features of MD without membrane instability (10). These mice exhibited enhanced store-operated calcium entry (SOCE) that is also observed in other models of MD due to mutations in DGC components (10). Moreover, this same study also demonstrated that inhibition of endogenous TRPC channels with a dominant negative encoding TRPC6 transgene could significantly attenuate the dystrophic phenotype in 2 separate mouse models of MD (10). Iwata et al. also reported that inhibition of $\mathrm{Ca}^{2+}$ influx via TRPV channels can mitigate the dystrophic 
A

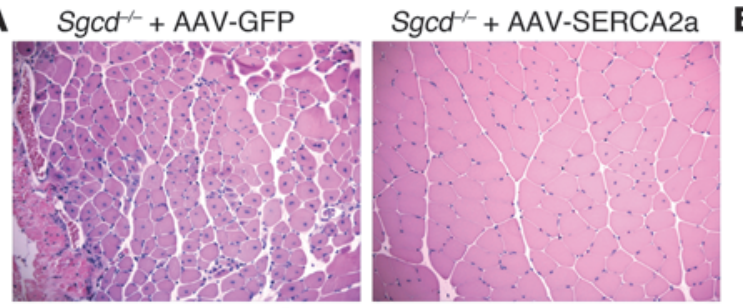

Gastrocnemius

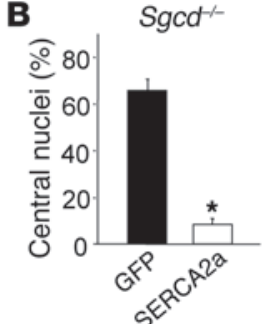

\section{Figure 8}

AAV9-SERCA2a gene therapy mitigates disease in $\mathrm{SgCd}^{-/}$gastrocnemius. (A) Representative histological H\&E stain of gastrocnemius from injected neonates taken 6 weeks later given control AAV9-GFP or experimental AAV9-SERCA2a. Original magnification, $\times 200$. (B) Quantitation of myofiber central nucleation in the 2 groups taken from 4 mice analyzed. ${ }^{*} P<0.05$ versus GFP. phenotype (33). These data support the hypothesis that enhanced $\mathrm{Ca}^{2+}$ influx alone is sufficient to induce MD, either through membrane rupture events or activated channels. That the SERCA1 transgene dramatically attenuated dystrophic muscle disease in TRPC3 Tg mice not only basically proves that the effect is entirely $\mathrm{Ca}^{2+}$ dependent, but also strongly suggests that enhanced SR Ca ${ }^{2+}$ cycling and reuptake can directly counteract a membrane-leak effect in promoting myofiber degeneration.

Previous work performed in this laboratory demonstrated that increased cytosolic $\mathrm{Ca}^{2+}$ can promote mitochondrial matrix $\mathrm{Ca}^{2+}$ overload and necrotic cell death via MPTP opening events (14). For example, inhibition of MPTP opening by genetic deletion of cyclophilin D or use of Debio-025 prevented cell death and reduced the muscular disease phenotype in $\mathrm{Sgcd}^{-/}$, Lama2 $^{-/-}$, and $m d x$ mice (14). Consistent with a $\mathrm{Ca}^{2+}$-driven pathway to MPTP opening and cell death, we observed that mitochondria isolated from SERCA1/ $\mathrm{Sgcd} \mathrm{d}^{-/}$mice were protected from swelling that otherwise occurs in mitochondria isolated from $\mathrm{Sgcd}^{-/-}$muscle (Figure 7, A-C). In addition to activation of MPTP opening and calpain $(12,13)$, dysregulated $\mathrm{Ca}^{2+}$ entry can result in the generation of reactive oxygen and nitrogen species to further contribute to membrane instability and myofiber degeneration (34-36). Enhanced removal of $\mathrm{Ca}^{2+}$ with the SERCA1 transgene not only protected the mitochondria from swelling and reduced calpain activation, but also provided some degree of membrane protection as assayed by EBD uptake, suggesting that increased $\mathrm{Ca}^{2+}$ influx generates a "feed-forward" disease process that further weakens the sarcolemma.

Overexpression of SERCA1-enhanced skeletal muscle EC coupling, such as a significant decrease in resting $\mathrm{Ca}^{2+}$ levels, enhanced amplitude of the $\mathrm{Ca}^{2+}$ transient and faster $\mathrm{Ca}^{2+}$ clearance from the cytoplasm (Figure 1, D-F). Interestingly, cardiac overexpression of SERCA1 also resulted in similar enhancement in cardiac function at baseline (37). In dystrophic muscle, the kinetics of SR $\mathrm{Ca}^{2+}$ handling are likely depressed, potentially due to reduced SERCA activity $(16-18,38)$. Although, the mechanism of altered SERCA1 activity in MD is not currently known, we observed a 50\% increase in sarcolipin mRNA in the quadriceps of $\mathrm{Sgcd}^{-/-}$mice (data not shown). Sarcolipin is a small SR membrane protein that inhibits SERCA1 activity. Indeed, Campanaro et al. also reported a similar increase in sarcolipin mRNA expression in muscle obtained from dysferlinopathy patients (39).

It is also very interesting to note that muscles spared of disease in $m d x$ mice, the laryngeal muscles, show significant overexpression of SERCA1 with disease (40). More recently, in direct support of our study, AAV-SERCA1a gene transfer in $m d x$ mice was shown to decrease centrally located nuclei in the diaphragm and to reduce eccentric contraction-induced damage (41). This study was particularly interesting, as the beneficial effect of the viral therapy lasted out to 6 months of age, suggesting that such an approach could provide sustained protection in humans (41). In our hands, direct injection of AAV9-SERCA2a in the gastrocnemius produced an astonishing protection from dystrophic disease in $\mathrm{Sgcd}^{-/-}$mice (Figure 8), again suggesting an entirely novel approach for gene therapy in humans with MD.

The experimental approaches discussed above suggested the hypothesis that enhanced $\mathrm{Ca}^{2+}$ removal directly protects against MD. If $\mathrm{Ca}^{2+}$ really is the "final common pathway" for myofiber necrosis downstream of most genetic mutations that induce MD, a universal therapy might easily involve approaches that augment cytosolic $\mathrm{Ca}^{2+}$ clearance. Here we utilized SERCA1 overexpression to increase the rates of $\mathrm{Ca}^{2+}$ reuptake into the SR to possibly reduce the effects associated with chronic $\mathrm{Ca}^{2+}$ leak from activated channels and membrane ruptures. In heart failure, a similar defect in membrane stability and $\mathrm{Ca}^{2+}$ handling has been observed and shown to underlie progressive cell death and worsening of disease $(42,43)$. In this context, SERCA2 overexpression by transgenesis or gene therapeutic strategies, or disinhibition of endogenous SERCA2 by deletion of phospholamban, dramatically protected mice from heart failure. Indeed, results of a recent gene therapy trial in heart failure patients with AAV-SERCA2a was reported to be effective (44). Thus, a gene therapeutic strategy with SERCA1/2 AAV might represent a "universal" approach to mitigate MD due to many types of gene mutations by correcting a final common defect in $\mathrm{Ca}^{2+}$ handling that appears to underlie most forms of MD.

\section{Methods}

Animals. A modified human skeletal $\alpha$-actinin promoter (a gift from Edna C. Hardeman, University of Sydney, Sydney, Australia) was used to create $\mathrm{Tg}$ mice overexpressing the rat skeletal muscle isoform of SERCA1 (provided by Muthu Periasamy, The Ohio State University, Columbus, Ohio, USA). Twelve Tg lines were generated, out of which 2 lines were analyzed for experiments. $S g \mathrm{Cd}^{-/-}$and TRPC3 Tg mice were described previously $(10,24)$. Use of animals in this study was approved by the IACUC at the Cincinnati Children's Hospital.

Mitochondrial isolation and swelling assay. Mitochondria from adult skeletal muscle hind limbs were isolated as described previously (45). Briefly, mice were sacrificed and hind limb skeletal muscle was removed, minced, and placed in homogenization buffer supplemented with $1 \mathrm{mg} / \mathrm{ml}$ trypsin, stirred for 30 minutes at $4^{\circ} \mathrm{C}$, and washed and homogenized in a glassteflon homogenizer. The muscle extract was then subjected to differential centrifugation to isolate mitochondria. Light scattering was measured using $250 \mu \mathrm{g}$ of mitochondria suspended in $1 \mathrm{ml}$ of buffer, and $200 \mu \mathrm{M}$ $\mathrm{CaCl}_{2}$ or $5 \%$ PEG (w/v) was used to induce mitochondrial swelling and shrinking, respectively. Change in absorbance at $540 \mathrm{~nm}$ was recorded every 10 seconds for up to 10 minutes.

Western blotting. Muscles were isolated, and extracts were prepared by homogenization in lysis buffer containing $50 \mathrm{mM}$ Tris, $\mathrm{pH}$ 7.4, $150 \mathrm{mM}$ $\mathrm{NaCl}$, and $1 \mathrm{mM}$ EDTA. Extracts were centrifuged at 13,000 $\mathrm{g}$ for 10 minutes, 
and $5-25 \mu \mathrm{g}$ of protein was separated on SDS- $8 \%$ polyacrylamide gels for Western blotting and chemiluminescent detection (Amersham Bioscience). SERCA1 was detected using mouse monoclonal antibody (1:2500) from Affinity Bioreagents.

Histological analysis. Muscles were paraffin embedded, and 7- $\mu \mathrm{M}$ histological sections were cut at the center of the muscle and stained with either H\&E or Masson's trichrome. Interstitial fibrotic regions were quantified using metamorph analysis of the percentage of blue area in Masson's trichrome sections.

Calpain activity assay. Calpain activity from quadriceps was assessed using a manufacturer-recommended protocol (Abcam). In brief, muscles were lysed in extraction buffer and spun at $20,817 \mathrm{~g}$ to generate supernatant for analysis. Then $250 \mu \mathrm{g}$ of protein was incubated in calpain substrate for 1 hour at $37^{\circ} \mathrm{C}$. Samples were read in triplicates in a plate reader equipped with a 400-nm excitation filter and 505-nm emission filter. Both positive and negative controls were performed as suggested by the manufacturer.

$E B D$ uptake. Animals were placed in cages fitted with running wheels for 5 days of voluntary running (all mice ran equivalent rotations per day). On the fifth day, mice were injected with $\mathrm{EBD}(10 \mathrm{mg} / \mathrm{ml}, 0.1 \mathrm{ml} / 10 \mathrm{~g}$ body weight $)$ and allowed to run for 48 additional hours. Mice were then sacrificed and quadriceps, TA, diaphragm, soleus, and gastrocnemius were embedded in OCT and snap frozen in liquid nitrogen for viewing as previously described (14).

$S R \mathrm{Ca}^{2+}$ uptake. Three-month-old mice were sacrificed, and the quadriceps were surgically removed and immediately homogenized in $50 \mathrm{mM}$ $\mathrm{KH}_{2} \mathrm{PO}_{4}$ (pH 7.0), $10 \mathrm{mM} \mathrm{NaF}, 1 \mathrm{mM}$ EDTA, $0.3 \mathrm{M}$ sucrose, $0.3 \mathrm{mM}$ phenylmethylsulfonylfluoride, and $0.5 \mathrm{mM}$ dithiothreitol. $\mathrm{Ca}^{45}$ uptake in muscle homogenates $(0.1 \mathrm{mg} / \mathrm{ml})$ was measured by a modification of the Millipore filtration technique as previously described (46). The rates of $\mathrm{Ca}^{2+}$ uptake were calculated by least-squares linear regression analysis at 30-, 60-, and 90 -second values of $\mathrm{Ca}^{2+}$ uptake. The data were analyzed by nonlinear regression using ORIGIN (version 6.0) software.

Measurement of $\mathrm{Ca}^{2+}$ transients. FDB fibers were isolated as previously described (47) and equilibrated in normal Ringer solution (140 mM NaCl, $4 \mathrm{mM} \mathrm{KCl}_{2} 2 \mathrm{mM} \mathrm{CaCl}_{2}, 1 \mathrm{mM} \mathrm{MgCl}_{2}, 10 \mathrm{mM}$ HEPES, $\mathrm{pH}$ to 7.4) for approximately 15 minutes. Fibers were loaded with $5 \mu \mathrm{M}$ Fura- 2 AM by incubating at room temperature for 45 minutes. $\mathrm{Ca}^{2+}$ transients were evoked via field stimulation using platinum electrodes at a stimulation frequency of $0.2 \mathrm{~Hz}$. The peak of the electrically evoked $\mathrm{Ca}^{2+}$ transient was recorded. The decay phase of the $\mathrm{Ca}^{2+}$ transient was analyzed as a parameter of $\mathrm{Ca}^{2+}$ reuptake from the cytosol. Fura 2-AM excitation ratio $(340 / 380 \mathrm{~nm})$ was recorded on a Nikon Ti-U inverted microscope equipped with a photomultiplier tube from Photon Technology Inc. Locally applied $500 \mu \mathrm{M} 4-\mathrm{CMC}$ bolus was used to assess RyRsensitive $\mathrm{SR} \mathrm{Ca}^{2+}$ load at the end of a train of electrically evoked $\mathrm{Ca}^{2+}$ transients. The peak 4-CMC response was analyzed to determine SR $\mathrm{Ca}^{2+}$ load.

Forced treadmill running. Three-month-old mice were placed in individual lanes of an electrically driven 4-lane treadmill (Omni-Pacer LC4/M; Colum- bus Instruments International) at the speed of $6 \mathrm{~m} / \mathrm{min}$ for 3 minutes. The treadmill measured 19 inches wide by 20 inches in length, with a conveyortype belt for running lanes measuring 3 inches wide by 12 inches long. A training regimen was first instituted for 10 minutes to familiarize them with the environment and shock grids adjustable from 0-2.0 mA. The speed was increased in increments of $2 \mathrm{~m} / \mathrm{min}$ every 3 minutes to a maximum speed of $18 \mathrm{~m} / \mathrm{min}$. Exhaustion was assessed as greater than 5 consecutive seconds on the shock grid without attempting to reengage the treadmill. Time spent on the treadmill before exhaustion or time to complete the protocol was recorded as average maximum time spent in exercise. The treadmill regimen was performed from the flat position (no incline or decline).

AAV9 production and injection. AAV9-CMV-GFP and AAV9-CMV-SERCA2a were produced using the 2-plasmid protocol and were grown in triple flasks for 24 hours (DMEM, 10\% FBS) prior to adding the calcium phosphate precipitate (48). After 72 hours, the virus was purified from benzonase-treated cell crude lysates over an iodixanol density gradient (OptiPrep; Greiner Bio-One Inc.), followed by heparin-agarose type I affinity chromatography (Sigma-Aldrich). Finally, viruses were concentrated and formulated into lactated Ringer's solution (Baxter Healthcare Corp.) using Vivaspin 20 Centrifugal concentrators $50 \mathrm{~K} \mathrm{MWCO} \mathrm{(Vivascience} \mathrm{Inc.)} \mathrm{and} \mathrm{stored} \mathrm{at}-80^{\circ} \mathrm{C}$. AAV9-SERCA2a and AAV9-GFP were injected into the left and right gastrocnemius, respectively, of 3-day-old $\mathrm{Sgcd}^{-/}$mice, amounting to approximately $10^{10}$ viral particles. Mice were sacrificed at 6 weeks and the gastrocnemius from both sides was processed, embedded, sectioned, and stained with $\mathrm{H} \& \mathrm{E}$, or cryosections were generated for GFP fluorescence assessment.

Statistics. All results are presented as mean \pm SEM. Statistical analysis was performed with unpaired 2-tailed $t$ test (for 2 groups) and 1-way ANOVA with Bonferroni correction (for groups of 3 or more). $P$ values less than 0.05 were considered significant.

\section{Acknowledgments}

This work was supported by grants from the NIH (to J.D. Molkentin, R.J. Hajjar, and E.G. Kranias). J.D. Molkentin was also supported by grants from the Jain Foundation and the Howard Hughes Medical Institute. S.A. Goonasekera was supported by a local affiliate American Heart Association grant.

Received for publication May 27, 2010, and accepted in revised form December 1, 2010.

Address correspondence to: Jeffery D. Molkentin, Cincinnati Children's Hospital Medical Center, Howard Hughes Medical Institute, Molecular Cardiovascular Biology, 240 Albert Sabin Way, MLC 7020, Cincinnati, Ohio 45229, USA. Phone: 513.636.3557; Fax: 513.636.5958; E-mail: jeff.molkentin@cchmc.org.
1. Muir LA, Chamberlain JS. Emerging strategies for cell and gene therapy of the muscular dystrophies. Expert Rev Mol Med. 2009;11:e18.

2. Kaplan JC. Gene table of monogenic neuromuscular disorders (nuclear genome only). Neuromuscul Disord. 2009;19(1):77-98.

3. Allen DG, Gervasio OL, Yeung EW, Whitehead NP. Calcium and the damage pathways in muscular dystrophy. Can J Physiol Pharmacol. 2010;88(2):83-91.

4. Fong PY, Turner PR, Denetclaw WF, Steinhardt RA. Increased activity of calcium leak channels in myotubes of Duchenne human and $\mathrm{mdx}$ mouse origin. Science. 1990;250(4981):673-676.

5. Fraysse B, et al. The alteration of calcium homeostasis in adult dystrophic mdx muscle fibers is worsened by a chronic exercise in vivo. Neurobiol Dis. 2004;17(2):144-154.

6. Turner PR, Fong PY, Denetclaw WF, Steinhardt
RA. Increased calcium influx in dystrophic muscle. J Cell Biol. 1991;115(6):1701-1712.

7. Turner PR, Westwood T, Regen CM, Steinhardt RA. Increased protein degradation results from elevated free calcium levels found in muscle from mdx mice. Nature. 1988;335(6192):735-738.

8. De Backer F, Vandebrouck C, Gailly P, Gillis JM. Longterm study of $\mathrm{Ca}(2+)$ homeostasis and of survival in collagenase-isolated muscle fibres from normal and mdx mice.J Physiol. 2002;542(pt 3):855-865.

9. Gailly P, Boland B, Himpens B, Casteels R, Gillis JM. Critical evaluation of cytosolic calcium determination in resting muscle fibres from normal and dystrophic (mdx) mice. Cell Calcium. 1993; 14(6):473-483.

10. Millay DP, Goonasekera SA, Sargent MA, Maillet $\mathrm{M}$, Aronow BJ, Molkentin JD. Calcium influx is sufficient to induce muscular dystrophy through a TRPC-dependent mechanism. Proc Natl Acad Sci US A. 2009;106(45):19023-19028.

11. Whitehead NP, Yeung EW, Allen DG. Muscle damage in $\mathrm{mdx}$ (dystrophic) mice: role of calcium and reactive oxygen species. Clin Exp Pharmacol Physiol. 2006;33(7):657-662.

12. Spencer MJ, Croall DE, Tidball JG. Calpains are activated in necrotic fibers from $\mathrm{mdx}$ dystrophic mice. J Biol Chem. 1995;270(18):10909-10914.

13. Spencer MJ, Mellgren RL. Overexpression of a calpastatin transgene in $\mathrm{mdx}$ muscle reduces dystrophic pathology. Hum Mol Genet. 2002;11(21):2645-2655.

14. Millay DP, et al. Genetic and pharmacologic inhibition of mitochondrial-dependent necrosis attenuates muscular dystrophy. Nat Med. 2008;14(4):442-447.

15. Rossi AE, Dirksen RT. Sarcoplasmic reticulum: the dynamic calcium governor of muscle. Muscle Nerve. 2006;33(6):715-731. 
16. Divet A, Huchet-Cadiou C. Sarcoplasmic reticulum function in slow- and fast-twitch skeletal muscles from mdx mice. Pflugers Arch. 2002;444(5):634-643.

17. Divet A, Lompre AM, Huchet-Cadiou C. Effect of cyclopiazonic acid, an inhibitor of the sarcoplasmic reticulum Ca-ATPase, on skeletal muscles from normal and mdx mice. Acta Physiol Scand. 2005; 184(3):173-186.

18. Kargacin ME, Kargacin GJ. The sarcoplasmic reticulum calcium pump is functionally altered in dystrophic muscle. Biochim Biophys Acta. 1996;1290(1):4-8.

19. Bellinger AM, et al. Hypernitrosylated ryanodine receptor calcium release channels are leaky in dystrophic muscle. Nat Med. 2009;15(3):325-330.

20. Fauconnier J, et al. Leaky RyR2 trigger ventricular arrhythmias in Duchenne muscular dystrophy. Proc Natl Acad Sci U S A. 2010;107(4):1559-1564.

21. Allen DG, Gervasio OL, Yeung EW, Whitehead NP. Calcium and the damage pathways in muscular dystrophy. Can J Physiol Pharmacol. 2010;88(2):83-91.

22. Deconinck N, Dan B. Pathophysiology of duchenne muscular dystrophy: current hypotheses. Pediatr Neurol. 2007;36(1):1-7.

23. Durbeej M, Campbell KP. Muscular dystrophies involving the dystrophin-glycoprotein complex: an overview of current mouse models. Curr Opin Genet Dev. 2002;12(3):349-361.

24. Hack AA, et al. Differential requirement for individual sarcoglycans and dystrophin in the assembly and function of the dystrophin-glycoprotein complex. J Cell Sci. 2000;113(pt 14):2535-2544.

25. Hollingworth S, Zeiger U, Baylor SM. Comparison of the myoplasmic calcium transient elicited by an action potential in intact fibres of $\mathrm{mdx}$ and normal mice. J Physiol. 2008;586(pt 21):5063-5075.

26. DiFranco M, Woods CE, Capote J, Vergara JL. Dystrophic skeletal muscle fibers display alterations at the level of calcium microdomains. Proc Natl Acad Sci US A. 2008;105(38):14698-14703.

27. Woods CE, Novo D, DiFranco M, Vergara JL. The action potential-evoked sarcoplasmic reticulum calcium release is impaired in $\mathrm{mdx}$ mouse muscle fibres. J Physiol. 2004;557(pt 1):59-75.

28. Ervasti JM, Sonnemann KJ. Biology of the striated muscle dystrophin-glycoprotein complex. Int Rev Cytol. 2008;265:191-225.

29. Turk R, et al. Common pathological mechanisms in mouse models for muscular dystrophies. FASEB J. 2006;20(1):127-129.

30. Gissel H. The role of $\mathrm{Ca} 2+$ in muscle cell damage. Ann N Y Acad Sci. 2005;1066:166-180.

31. Cohn RD, Durbeej M, Moore SA, Coral-Vazquez R, Prouty S, Campbell KP. Prevention of cardiomyopathy in mouse models lacking the smooth muscle sarcoglycan-sarcospan complex. J Clin Invest. 2001;107(2):R1-R7.

32. Hoshijima M, et al. Chronic suppression of heartfailure progression by a pseudophosphorylated mutant of phospholamban via in vivo cardiac rAAV gene delivery. Nat Med. 2002;8(8):864-871.

33. Iwata Y, Katanosaka Y, Arai Y, Shigekawa M, Wakabayashi S. Dominant-negative inhibition of $\mathrm{Ca} 2+$ influx via TRPV2 ameliorates muscular dystrophy in animal models. Hum Mol Genet. 2009;18(5):824-834.

34. Durham WJ, et al. RyR1 S-nitrosylation underlies environmental heat stroke and sudden death in Y522S RyR1 knockin mice. Cell. 2008;133(1):53-65.

35. Gervasio OL, Whitehead NP, Yeung EW, Phillips WD, Allen DG. TRPC1 binds to caveolin-3 and is regulated by Src kinase - role in Duchenne muscular dystrophy. J Cell Sci. 2008;121(pt 13):2246-2255

36. Whitehead NP, Pham C, Gervasio OL, Allen DG. $\mathrm{N}$-Acetylcysteine ameliorates skeletal muscle pathophysiology in $\mathrm{mdx}$ mice. J Physiol. 2008; 586(7):2003-2014.

37. Talukder MA, et al. Expression of SERCA isoform with faster $\mathrm{Ca} 2+$ transport properties improves postischemic cardiac function and $\mathrm{Ca} 2+$ handling and decreases myocardial infarction. Am J Physiol Heart Circ Physiol. 2007;293(4):H2418-H2428.

38. Tutdibi O, Brinkmeier H, Rudel R, Fohr KJ. Increased calcium entry into dystrophin-deficient muscle fibres of MDX and ADR-MDX mice is reduced by ion channel blockers. J Physiol. 1999;515(pt 3):859-868.
39. Campanaro S, et al. Gene expression profiling in dysferlinopathies using a dedicated muscle microarray. Hum Mol Genet. 2002;11(26):3283-3298.

40. Ferretti R, Marques MJ, Pertille A, Santo Neto H. Sarcoplasmic-endoplasmic-reticulum Ca2+-ATPase and calsequestrin are overexpressed in spared intrinsic laryngeal muscles of dystrophin-deficient mdx mice. Muscle Nerve. 2009;39(5):609-615.

41. Morine K, Sleeper MM, Barton ER, Sweeney L. Overexpression of SERCA1a in the $\mathrm{mdx}$ diaphragm reduces susceptibility to contraction induced damage [published online ahead of print June 11, 2010]. Hum Gene Ther. doi: 10.1089/hum.2010.077.

42. Kranias EG, Bers DM. Calcium and cardiomyopathies. Subcell Biochem. 2007;45:523-537.

43. Townsend D, et al. Chronic administration of membrane sealant prevents severe cardiac injury and ventricular dilatation in dystrophic dogs. JClin Invest. 2010;120(4):1140-1150.

44. Celladon Corp. announces that MYDICAR meets primary endpoint in phase 2 trial for treatment of advanced heart failure [press release]. La Jolla, California, USA: Celladon Corp.; April 28, 2010. http://www.celladon.net/index.php?option $=\mathrm{com}_{-}$ content $\&$ view $=$ article $\&$ id $=74 \&$ Itemid $=88$.

45. Frezza C, Cipolat S, Scorrano L. Organelle isolation: functional mitochondria from mouse liver, muscle and cultured fibroblasts. Nat Protoc. 2007; 2(2):287-295

46. Slack JP, Grupp IL, Ferguson DG, Rosenthal N, Kranias EG. Ectopic expression of phospholamban in fast-twitch skeletal muscle alters sarcoplasmic reticulum $\mathrm{Ca} 2+$ transport and muscle relaxation. J Biol Chem. 1997;272(30):18862-18868.

47. Lueck JD, et al. Chloride channelopathy in myotonic dystrophy resulting from loss of posttranscriptional regulation for CLCN1. Am J Physiol Cell Physiol. 2007;292(4):C1291-C1297.

48. Suckau L, et al. Long-term cardiac-targeted RNA interference for the treatment of heart failure restores cardiac function and reduces pathological hypertrophy. Circulation. 2009;119(9):1241-1252. 